\title{
The Essence of Simulation and Artificial Intelligence
}

\author{
Tianjin Feng \\ Department of Electronic Engineering, Ocean University of China, Qingdao, P. R. China
}

\section{Email address:}

tjfeng@mail.ouc.edu.cn

\section{To cite this article:}

Tianjin Feng. The Essence of Simulation and Artificial Intelligence. American Journal of Electrical and Computer Engineering. Vol. 3, No. 1, 2019, pp. 10-19. doi: 10.11648/j.ajece.20190301.12

Received: December 26, 2018; Accepted: April 2, 2019; Published: June 18, 2019

\begin{abstract}
In response to the controversy over whether machines can think and the queries on artificial intelligence (AI), this paper clarifies that the essence of simulation and AI (i.e. simulation intelligence) should be pursuing assimilation (similartaxis) as well as keeping dissimilation to a prototype (natural intelligence). Both commonness and obvious otherness (individuality) must exist between them. This is a 'Both/And' thinking mode and not a simplified exclusive 'Either/Or' thinking. Based on four definitions and five hypotheses, some corollaries are educed logically to clarify the relation between machine intelligence and human mind and to answer the queries on AI. This paper points out that the 'Either/Or' thinking, such as 'either true intelligence or false' or 'either whole mind or nothing', resulted in some cognitive mistakes on AI and the mysticism had most deleterious consequences among the research of AI and cognitive science.
\end{abstract}

Keywords: Simulation, Artificial Intelligence, Thinking Mode, Consciousness, Spirit

\section{Introduction}

British mathematician, natural philosopher and AI father Alan Turing (1912-1954) answered affirmatively the question "Can machines think?" and defended the feasibility of AI in his famous article "Computing machinery and intelligence" [1, 2]. It is worth noting that S. Russell \& P. Norvig write (2003) "Turing examined a wide variety of possible objections to the possibility of intelligent machines, including virtually all of those that have been raised in the half century since his paper appeared. [3]"

In recent decades, some philosophers and cognitive scientists with divergent interests respectively answer such questions as: Are human intelligence and machine intelligence the same? Can a machine have a mind, mental states, and consciousness in the same way that a human being can? The nature of the debate surrounding these questions is the same with the primary debate.

The author of this paper has been concerned about the debates since 1970's and identified that there are two inverse thoughts in it [4]. For the time being, they are called the one side and the opposite side. The one side highlights the intercommunity between human being and machines, and the opposite side highlights the essential difference between them and infeasibility of AI.
The one side inherits the following thought chain: Socrates was asking an algorithm to distinguish piety from non-piety (around 450 B. C.). Aristotle went on to try to formulate more precisely the laws governing the rational part of the mind [3]. Descartes (1596-1650) conceived of animals as automata; Pascal (1623-1662) and Leibniz (1646-1716) attempted to create a logical calculus of all human ideas; and La Mettrie (1709-1751) wrote a book entitled 'L' Homme Machine or Man a Machine". Turing built a machine (1936), Universal Turing Machines, to model human's mind. As the founder of AI discipline, M. Minsky (1927-2016) said that the brain happens to be a meat machine.

If the one side argument has the characteristics of scientific hypothesis, then the opposite side has more characteristics of belief, and has a deeper historical origin. Interpreting the nature of the mind was the "patent" of philosophy and theology since ancient times, but seems to have little to do with science. The once dominant view was that only human beings possessed the mind, and machines possessed only simple, mechanical operations or calculations, at most a bit like, rather than real thinking, and could not be conscious and spiritual. Many theologians censure that a machine may have intelligence. There is a special section, "The Theological Objection", in Turing's article [1]. He wrote: they believe that "Thinking is a function of man's immortal soul. God has given 
an immortal soul to every man and woman, but not to any other animal or to machines. Hence no animal or machine can think." "I am unable to accept any part of this."

The debates have promoted prosperity and cooperation of philosophy, psychology, neuroscience, computer sciences and cognitive science. On the other hand, a simplified thinking pattern, such as either identical or nothing, creates some useless debates and logical mistakes. It seems to say that if machine intelligence is not exactly the same (identical) with human intelligence, it should be not intelligent (nothing). It has deviate from right direction of science.

It is most desirable to establish a widely accepted theoretical foundation for the relation between AI and biological intelligence. Article [5] studied to get over the confusion and logical mistakes based on some clear-cut concepts about "simulation". This article studies the general process of the creation and evolvement of $\mathrm{AI}$ and points out that $\mathrm{AI}$ is the result of convergence and alienation of natural intelligence. Based on four definitions and five hypotheses, some inferences are educed to prove or disprove some arguments and to reveal the essence of AI.

For convenience, here are some symbols in the set theoretic language as follows. The set B denotes biological systems, set $b(i)$ denotes $\mathrm{BI}$ and is a subset of $\mathrm{B}$, and $b(i, k)$ is a kind of $b(i)$ and a subset of $b(i)$. The set $\mathrm{M}$ denotes the machines, set $m(i)$ denotes machine intelligence (AI) and is a subset of $\mathrm{M}$, and $m(i, k)$ is a kind of $m(i)$ and a subset of $m(i)$.

\section{Simulation: Convergence and Alienation}

As everyone knows that simulation is a widely applied concept, but it is strictly not clear in meaning. Usually, the concept of "simulation" is not rigorous that is one of the reasons leading to some confusion in the debate on AI.

\subsection{The Essence of Simulation}

Definition 1. The simulation is to design or construct an artifact $M$ in a different physical system (alienation) contrasting to the original $B$, which achieves certain similar characteristics or behaviors of $B$ (convergence).

Simulation can focus on the behavior or internal structure of $\mathrm{B}$, and consider both behavior and structure.

Convergence, that is to explore and achieve some commonality between $\mathrm{M}$ and $\mathrm{B}$, is the primary goal of simulation. Alienation means that the realization of a certain structure or function of B by a different physical system M will inevitably bring into play the characteristics and advantages of $\mathrm{M}$ itself, and the differences between $\mathrm{M}$ and $\mathrm{B}$ will also appear. It means that a simulation $M$ is the result of convergence and alienation of archetype B, and both commonality and obvious differences between $\mathrm{M}$ and $\mathrm{B}$ must exist. This is the essence of simulation with a 'both/and' and not an exclusive 'either/or' thinking mode.

Simulation is closely related to modeling. Modeling is the mathematical and/or physical approximation of the prototypes of things. A model can be an abstraction or generalization of reality within a clearly defined area of $B$ to get a representation of its inherent rules or information processing mechanisms. So, modeling is a way used to get the commonalities of $\mathrm{M}$ and $\mathrm{B}$, and to construct an artifact $\mathrm{M}$ to simulate B. Obviously, modeling and simulation are important means and main contents of science and technology. If there is no modeling, there will be no science. For example, the laws of physics are models of the physical world. Modeling has been applied to modern psychology and neuroscience (as described in [6] and [7]).

The founder of cybernetics Wiener (1894-1964) reveals the commonalities between animals and machines in control, communication and signal processing [8]. This idea has been expanded. Bioinformatics holds that the distance between carbon-based bioinformatics and silicon-based electronic information processing has been rapidly shortened, both conceptually and practically. (such as outlined in [9]) On the other hand, alienation means that there must be differences between $\mathrm{M}$ and $\mathrm{B}$. Wiener said humorously that the best physical model of a cat is also a cat, preferably the cat itself. Von Neumann (1903-1957), the father of modern computers, believes that both humans and computers are "automata" (generalities), and that there are significant differences in the components, structures and working mechanisms of the two to achieve generalities [10].

A major difference between $\mathrm{M}$ and $\mathrm{B}$ is that $\mathrm{B}$ or human intelligence is the object of study, and people always try to find something about it. But there is always something $(x)$ unknown to be explored in B (whether it can be exhausted is not discussed here). Because one can not simulate the unknown $x$, it brings people mystery and is the soil of mysticism. On the contrary, $\mathrm{M}$ is a human creation (artifact, intelligent machine) based on known knowledge.

It is interesting that the difference between $\mathrm{B}$ and $\mathrm{M}$ formed by unknown $x$ has a subtle nature: it is not clearly described; once it is described in detail, the unknown is transformed into known, and simulation (or partial simulation) is possible, and the difference disappears to some extent. So, the difference and $x$ are dynamic.

The aims of simulation could be approximately divided into two. One is that the simulation system M is means to research and understand the original $\mathrm{B}$. Another is to get a novelty and more powerful implement $M$ for technical applications. Naturalness, the two aims may be concurrent in some research. For the second aim, the functions and mechanism of $\mathrm{B}$ is edification for design $\mathrm{M}$, and the coherence of $\mathrm{M}$ and $\mathrm{B}$ is not goal with sedulous care. It is essentially stressed that an applied system $\mathrm{M}$ is not possible to be only imitative and must be a productive system with many particular engineering attributes $m p$ (p: particular) that B may not hold, i.e.

$$
\forall m p(M \cap \bar{B}) \text { which just like } \forall b p(B \cap \bar{M})
$$

Similarly, there are many physiological properties $b p$ of B that $\mathrm{M}$ does not possess. 
Consequently, the developing of M must pass through itself route, which is not a copy of $\mathrm{B}$. Some machine learning algorithms, such as error back-propagation (BP), support vector machines (SVM), etc., are all based on the traditional or statistic mathematics rather than through simulation of biological processes. That is to say, convergence should not be pursued blindly. The $m p$ and $b p$ may lead to the uniqueness or superiority of $\mathrm{M}$ and $\mathrm{B}$, respectively.

\subsection{What is $A I$}

Having defined the essence of "simulation", then there is the basis for clarifying the nature of AI. For the sake of the convenience of discussion, a definition of intelligence is given here, which has widely been accepted (such as in [3] and [11]).

Definition 2. Intelligence is an ability to adapt successfully to the environment and find effectively satisfying solutions of real problems based on past experience.

It is equally applicable to human being, biological system, machine, consciously intelligence, unconsciously intelligence, biological groups and evolutionary processes. A satisfying solution is a general concept for many complicated problems, for which an optimal solution may not exist or doesn't make sense. This definition of intelligence embodies an idea that memory (involved past experience) and the ability of prediction (involved how to adapt oneself) is the base of general intelligence.

Based on the idea that AI is simulation intelligence, this paper gives the definition of AI similar to definition 1 , as follows.

Definition 3. AI is to construct an artifact $m(i)$ in a physical system different from (alienate) the biologically prototype $b(i)$, which achieves certain similar intelligent behaviors (convergence) of $b(i)$.

Similar to general simulation, AI can also focus on simulating the biological intelligence behavior, or the internal structure and behavior of the nervous system, or both. Turing focused on human intelligence behavior simulation, so he used "polite Convention" to define AI: "If a machine acts as intelligently as a human being, then it is as intelligent as a human being.[1]" In like manner, artificial consciousness (AC) can be considered that makes machine's behave like people's conscious behavior.

The Definition 3 means that AI is the result of convergence and alienation of BI, so that both commonality and obvious differences between AI and its biologically prototype must exist. This is the essence of AI with a 'both/and' and not an exclusive 'either/or' thinking mode. A typical example of 'either/or' thinking mode is that AI must be "either complete or meaningless" relative to its biological prototype (See "Reasoning 4" in this article).

\section{Five hypotheses}

Based on the previous discussion of the nature of simulation and $\mathrm{AI}$, this paper summarizes the following five hypotheses as the basis for consensus and inference.

1) The approximate separability hypothesis of the whole:
Any complex thing (or system) can be approximately regarded as composed of some relatively (non-absolutely) independent parts, subsystems or hierarchies.

The approximate separability of things is the foundation of all human activities and science. Only when the world is approximately divided into different fields and levels can it be possible to establish various disciplines such as physics, chemistry, psychology and so on. Every practical human activity is carried out in a specific aspect of things. People who ignore quantum mechanics (on the microscopic level of matter) can build buildings freely.

Modularization of human intelligence and cognition, and the hierarchical structure of cerebral cortex have been recognized. On the contrary, the idea that thinking can not be studied without emotion violates the fact and scientific spirit and hinders the development of artificial intelligence.

2) Complex nonlinear correlation hypothesis between part and whole: The whole has some attributes that its parts do not possess. Some overall complexities emerge (generate) in its various parts of nonlinear interaction; complexity comes from simplicity; the parts may also have an attribute related to an attribute of the whole.

This hypothesis holds that the idea of absolute separability of the whole and reductionism make the mistake of ignoring the complex interaction between parts. For example, individual neurons do not possess the overall mental state and function of emotion, language and consciousness. They are "emerging" brain functions in the interaction of numerous neurons, several neural networks, and the neurodynamics of the brain's overall system. On the other hand, since the mid-20th century, neuroscientists have discovered that brain neurons possess perceptual and learning [14] and pattern recognition abilities [15], and are associated with feelings, learning and consciousness at the higher levels of the brain system, i.e. there is a "neural correlation of consciousness (NCC)".

3) Simulation feasibility hypothesis: If and only if a BI behavior or mechanism is known (can be precisely described, such as formal coding), it is possible to develop a simulation intelligence.

This assertion was printed in the program for the Dartmouth Conference of 1956, widely considered the "birth of AI." The Dartmouth proposal: "Every aspect of learning or any other feature of intelligence can be so precisely described that a machine can be made to simulate it.[3]"

4) Unknown x existence hypothesis: As a research object, BI contains some unknown factors $x$ which can not be simulated, and $x$ would change dynamically with the progress of human cognition.

$\mathrm{BI}$ includes unknown factors (such as intuition, premonition and consciousness) that give us a sense of mystery. This is the biggest difference between AI and BI. However, the purpose of science is to change the unknown into the known, so that the known areas continue to expand and the boundaries of the unknown continue to retreat.

5) Special attribute existence hypothesis: Based on different physical systems, AI and its BI prototypes must have some 
special attributes that do not exist with each other. These differences are dynamic.

To sum up the above and the approximate separability hypothesis of the whole, $b(i)$ may be considered as an integration of $b(i, k), b p(i, k) \quad(k=1,2, \cdots, n)$, and $x$. So, it could formally be summarized as follows:

$$
b(i)=\phi\left(\bigcup_{k=1}^{n}[b(i, k) \bigcup b p(i, k)] \bigcup x\right)
$$

And

$$
\forall b p(i, k) \bigcup x[b(i, k) \bigcap \overline{m(i, k)}]
$$

Where $\phi$ integrates and connects all of $b(i, k), b p(i, k)$, and $x$ into a whole $b(i)$. Among them, the special attributes $b p(i, k)$ and unknown factors $x$ have nothing to do with machine intelligence.

Correspondingly, there may be an AI product $m(i)$ would be gained as an integration of $m(i, k), m p(i, k)$, and some sealed $y$. Let $R\left(z_{1}, z_{2}\right)$ denote the common attributes $R$ of $z_{1}$ and $z_{2}$. Therefore, there are

$$
m(i)=g\left(\bigcup_{k=1}^{n}[R(m(i, k), b(i, k)) \bigcup m p(i, k)] \bigcup y\right)
$$

And

$$
\forall m p(i, k)[m(i, k) \cap \overline{b(i, k)}]
$$

Here, $R($, ) represents the result of the convergence (advolution) of $m(i, k)$ to $b(i, k)$.

These expressions are all qualitative or as of a conception. There is no a right method to describe a kind of $\mathrm{BI}, b(i, k)$, using mathematical variables or elements in set theory. According to modern brain science, the whole brain function is composed of several (n) modules, and the interpreter in the left brain is responsible for this integration $\phi$. But it is not clear how much $\mathrm{n}$ the function $\phi$ is. In contrast, $m(i)$ is a combination of $\mathrm{n}$ known modules $m(i, k)$, the function $g$ is known but may be complex, or may also be a complex system of human-computer interaction.

It is worth to say what some sealed factors $y$ exist in an artifact. For instance, there may be unknown defects (bugs) and viruses in computer systems. The internal behavior of artificial neural network (ANN) or self-organizing machine is obviously unpredictable and random (see [16]). Self- organizing and self-learning machines with random factors and chaos are bound to have many unknown properties and behaviors. This is one of the many problems of AI that we are going to face.

\section{A Number of Reasoning}

From the above definitions and hypotheses, the following deductions can be obtained.

Reasoning 1. Simulation is not duplication.

Prof. J. L. Casti invented a banquet in 1949 in Cambridge, England, in an interesting essay [23]. He invited A. Turing, philosopher L. Wittgenstein, quantum physicist E. Schrödinger and geneticist J. B. S. Haldane to discuss the possibility of AI. In the meantime, Turing said frankly, "machines may duplicate human brain and mental processes". Although this conclusion has not been seen in his official anthologies, similar ideas appear from time to time in the writings of some modern scholars.

The definition of simulation, the definition of AI, the hypothesis of unknown $x$ existence and the hypothesis of specific performance all show that simulation is not duplication, and that it is impossible for AI to perform exactly the same as its biological prototype. It is a wrong judgement that machines can duplicate the brain and the whole process of thinking. Similarly, the American philosopher Searle proposed the strong AI hypothesis (1999): "Computers properly programmed and with the correct input and output will therefore have ideas identical to those of humans. [3]" Such a "strong AI", which has "exactly the same idea as human beings", is impossible to achieve. In short, in terms of the relationship between $\mathrm{AI}$ and human intelligence, the concepts of "duplication", "exactly the same as" are wrong.

Reasoning 2. AI that does not fully depict human thought is worth affirming.

Wittgenstein, a famous contemporary philosopher of Turing, emphasized that the study of intelligence can not be separated from "a living person as a whole [13]" and from "the whole mind" (including emotion and consciousness [18, 19]), while the human mind is full of mystery, so intelligence can not be simulated. "AI is an unrealistic and meaningless utopia, because formal logic can not completely describe human thought" [17], wrote Ziman, a well-known scholar.

Such arguments criticize the errors of the "replicated (completely depicted) brain and mind", but go to the other extreme, adopting the wrong logic that "incomplete means meaningless"; and belong to the Either/Or mode of thinking that is "not the whole mind, not the intelligence". It rejects the approximate separability hypothesis of the whole and simulation feasibility hypothesis with entirety and unknown existence, fails to grasp the nature of convergence and alienation of "simulation", and deviates from the basis of scientific research.

Reasoning 3. One can't deny the intelligence of a system just because it needs human intervention.

Taking computer pattern recognition and biological classification as an example, Ziman pointed out that computer has no intelligence because it requires human intervention (for example, determining pattern features by human experience) [17]. This argument is also an example of "not integrity, not intelligence" (Either/Or).

Building intelligent systems with human intervention is a widely used method in the field of AI application [20]. A successful man-machine system not only reduces the workload, but also completes the original human intelligence 
work better. That is to say, the computer has overfulfilled some of human intelligent work. So, according to the definition of AI, computers are intelligent.

Reasoning 4. Human intelligence and AI may have the ability to outperform each other.

As everyone knows, modern science is constantly revealing the mysteries of life, brain, spirit, consciousness, emotion and intelligence, and creating new disciplines such as AI, cognitive science and artificial life. On the other hand, as confirmed by the unknown $x$ existence hypothesis and simulation feasibility hypothesis, there are still many unknowns in the field of biological intelligence, which can not be simulated at the moment. This is the main reason why human intelligence currently is far superior to AI in many aspects.

As affirmed by the special attribute existence hypothesis, it is an inevitable fact that after the alienation of biological intelligence into AI, relying on high-speed development and powerful modern science and technology, AI is far beyond human beings as the unique functions of computer systems, such as high-speed, stable computing and search capabilities. In general, this is also the goal pursued by human technology.

An interesting example of AI far exceeding mankind is that Ke Jie, the world champion of the Go world, said after the defeat of AlphaGo (quoted from Ko Jie's micro-blog, 2017): "After humanity spent thousands of years improving their tactics, computers tell us that humans are completely wrong". "I would go as far as to say not a single human has touched the edge of the truth of Go." This proves that AlphaGo has a unique strategy that surpasses that of human chess players, and finds some unknown regularity in Go.

Reasoning 5. Computers certainly are intelligent machines.

A fairly popular view is that only computers running high-level intelligent software are "intelligent machines," and generally computers are not intelligent, or simply acknowledge that they are "smart". This view is illogical. moreover, it is ridiculous to avoid "intelligence" with "smart", because the two meanings are basically the same.

It is well known that Boolean algebra originates from the simulation of thinking logic and is the basis of computer hardware and software; Turing machine is proposed as the model of human thinking; B. Pascal and W. Leibniz develop computer with the goal of thinking machine; Von Neumann structure of computer simulate the mode of human information processing. The basic principles and methods of computer operating systems, such as addressing memory, buffer registers, indirect addressing, interruption, time-sharing, programs, subroutines, serial, parallel and other design ideas, are derived from human daily life transaction processing skills. Therefore, according to the definition of AI, no matter the level of software, the computer is an intelligent machine.

Interestingly, in the 17th century terminology and theories such as the "thinking machine" came into being, and there was no blatant blame for them in nearly three centuries. Today, there is no reason to deny this basic concept.

Reasoning 6. Turing test is the correct concept of AI.

Turing proposed in his famous article [1] that testing is a feasible way to examine whether a computer can think. The test arranged the dialogues between the segregated interrogators and someone $\mathrm{B}$ and a machine $\mathrm{M}$ respectively. If M succeeds in simulating human's ability to talk so that the interrogator can't tell them apart through the dialogue, it should be said that $M$ is intelligent, without having to study other differences between $\mathrm{B}$ and $\mathrm{M}$, and whether they are conscious or not. This is so called as the Turing test.

AI's skeptics say machine conversation and poetry are just patchwork symbols, not emotions, and don't know what they mean. Turing argues that we all know what other people say and do, and that there is no evidence to confirm the inner state of mind (the underlying problems). The people engage in conversation but no one ever asks the question "can you think?" People usually follow a polite convention that everyone thinks, but does not ask others' inner state.

The Turing test extends this polite convention to machines: don't ask the question "can M think?" If a machine acts as intelligently as a human being, then it is intelligent and it should lay aside the mystery about consciousness for the moment. Turing declared in [1] that "I do not wish to give the impression that I think there is no mystery about consciousness.""But I do not think these mysteries necessarily need to be solved before we can answer the question (Can machines think?) with which we are concerned in this paper."

Turing test's idea is the correct basic concepts of AI and is consistent with the approximate separability hypothesis of the whole and the definition of AI. On today's vibrant internet, chatbots, which serve customers, have exceeded Turing's expectations in their ability to talk to people.

Turing tests are also applied skillfully in the industrial field. For example, we developed a product AI formulation system [20], in which a set of neuro-fuzzy models for sensory-quality evaluation were used to replace the work of sensory appraiser in some production processes. When people can't tell which group of values is given by the AI system and which group is given by the assessor, the AI system passes the Turing test.

Reasoning 7. The idea of negating its intelligence based on the simplicity of AI mechanism is a simple mechanical thinking.

When a computer doctor successfully communicated with a patient and passed the Turing test, the famous physicist R. Penrose commented: "Though this may give an eerie impression that the computer has some understanding, in fact it has none, and is merely following some fairly simple mechanical rules. [25]" Next he discussed the chess-playing computers, thinking that computer chess depends on simple, fast and accurate computing ability to win, and human chess players have a conscious "judgment" ability, can be more profound than the machine analysis. He believes that this difference is much more obvious when playing Eastern Go [25]. The idea is that computers will lose to chess players in the Go game. He did not expect that AlphaGo completely shattered this view.

The viewpoint of denying AI's intelligence on the basis of its simplicity of operation mechanism is contrary to the definition of intelligence, the definition of AI, and complex 
nonlinear correlation hypothesis between part and whole. It is a lack of understanding of the dialectical relationship between complexity and simplicity, and also is a simple and mechanical way of thinking. For example, AlphaGo's Deep-learning methods are based on ANN, and the artificial neurons that make up ANN are "simple", but AlphaGo conquered all the human Go players without any suspense. In fact, the basic action of neurons in the biological brain is "all-or-none", similar to the "1-or-0" [9, 15] in digital logic, is also simple, However, a great deal of neuron interaction has brought up the most mysterious human spirit. A more simple example is that the music is based on simple notes, but there is an endless stream of complex and beautiful music.

Reasoning 8. The "onion skin" metaphor reveals the confusion of the AI doubters.

The "skin-of-an-onion" analogy in Turing's article [1] is very vivid and interesting.

The pioneers of AI (Leibniz, Boolean, etc.) passionately constructed logic machines that embodied the logic of human thinking; however, later successors disagreed, saying that logic machines were merely simple mechanical operations. "This we say does not correspond to the real mind: it is a sort of skin which we must strip off if we are to find the real mind. But then in what remains we find a further skin to be stripped off" [1]. For example, pattern recognition and learning used to be considered the real intelligence these successors were looking for. But a few years later, machine learning has made great strides, and the successors of these successors have similar mentality, thinking that pattern recognition, machine chess and learning algorithms are also simple operations, but also "skin", not really intelligent!And so on.

It's a lot like "play on reliance": once the mental function is incredibly realized by the program, soon someone stops admiring that it contains real intelligence. Real intelligence always hides behind the AI. Turing asked: in this way, a layer of skin is removed and what is left behind? Maybe the last thing I saw was skin? "Proceeding in this way do we ever come to the 'real' mind, or do we eventually come to the skin which has nothing in it? In the latter case the whole mind is mechanical" [1]. Another scientist sneered at: what is real AI? It is always the mysterious thing that has not yet been realized.

Obviously, this is not the right logic of thinking. It is also an example of "Either/Or" a simple way of thinking that is to say "either complete soul or meaningless skin". As has been said before, an AI may be a sort of "skin" and must also embody some ingredients of real intelligence in it. The idea of logical consistency is that new discoveries in biological intelligence and AI continue to emerge, and the content of AI continues to enrich and advance. Scientific development is inheriting. Generally speaking, the contents of the earlier stage of science are simpler than later ones, but they are all "true" science, not "skins" to be abandoned, except for some errors.

Unfortunately, more than half a century later, the ideological tendencies analyzed in Turing's article [1] still have a great influence. As Turing said, there are potential psychological problems: "We like to believe that Man is in some subtle way superior to the rest of creation. It is best if he can be shown to be necessarily superior, for then there is no danger of him losing his commanding position. [1]" So they are afraid that machines can think.

Reasoning 9. AI is both "as if" and embodying some "real" intelligence.

It is still widely accepted that AI could possibly (as if) act intelligently, as a simulated intelligence, and that denying it is a "real" intelligence. For example, on August 6, 2018, the Wall Street Journal reported on a thematic debate: Can contemporary AI be "real intelligence" or "really thinking"?

This view is called "weak AI" by some philosophers [3]. It admits that simulation is feasible and AI is simulated intelligence, but opposes "simulated" to "real" and argues that AI can not "really thinking". This view negates the convergence nature and value of simulation with the difference of simulation, denies that there is "real" content in simulation; in essence, it also negates the feasibility of simulation. So, it begins with acknowledging the feasibility of simulation and comes to the result that negates the feasibility of simulation, which is logically self- contradictory.

The American philosopher J. Searle figuratively said: No one will agree that we will get wet when we simulate a storm by computer [18]. Obviously, he wrongly explained the essence of computer simulation of the storm. One of the main purposes of computer simulation of storms is to find out the (real) mechanism and atmospheric dynamic laws (convergence) of storms. Everyone knows that it's easy to get people wet, as in movies and TV shows where a storm is simulated by spraying water and blowing wind. But that's just an external representation of a storm and is meaningful for art but not science. It's not helpful to understand the inherent laws of nature that produce it.

So computer simulation looks like a natural process, and at the same time, it does embody something real about the process. Similarly, AI is both "as if" and "real" in some aspects of biological intelligence. This is also a "both/and" thinking mode.

Reasoning 10. The argument that "Computers can only do what people programmed, so that they are without intelligence." is a tautology.

The effective syllogism of this popular argument is: All programs are not intelligent (major premise), computers do things according to the program (minor premise), so the computer can not have intelligence (conclusion). Obviously, this is a tautology. "Computers are not intelligent" are equivalent to "Computer programs are not intelligent" here. The major premise negates AI and repeats it in the conclusion. Therefore, there is no value in argument. And it does not even explain why computer programs are not intelligent.

Reasoning 11. The zombie reasoning is an improper thought experiment.

Some philosophers have designed a thought experiment called "brain substitution" [26, 27], which uses IC chips to replace the brain neurons of the subjects. They hypothesized that: 1) the function of the chip was identical to that of the neurons; 2) neither was related to consciousness. After substitution, we get a zombie, which is indifferent from 
behavior but not conscious. They want to prove that even if the machine acts like human beings, it has no consciousness.

Since neurons and its analog chips all have NCC functions, they must also retain the physiological basis of consciousness generation when they replace each other, eventually, the complex neurodynamic effects of the brain system lead to the recovery (emergence) of consciousness.

From another perspective, one can ask: If the behavior of zombies without consciousness is really the same as that of conscious people, then what does consciousness mean to people? Why do humans have to be conscious? How do you know if the "real people" and "zombies" are with or without consciousness? Obviously, the idea that consciousness and behavior can be completely separated is absurd, and the idea of zombie is disordered in logic.

Reasoning 12. AI is a huge discipline and should not be given exclusiveness to a model.

Biological systems and their intelligence are highly complex and hierarchical. Therefore, AI must be a huge discipline arising from interdisciplinary interaction. In its development, it is harmful to identify a model as the only way to get AI.

In the 1980s and 1990s, there was a controversy between connectionism and symbolism in AI field, and both sides had utterances that totally negated each other. It turns out that they are two distinctive schools of thought. Symbolism and expert systems, connectionism and neural networks, evolutionary algorithms, etc. all may be a kind of simulation intelligence in a certain hierarchy. Connectionism attempts to simulate the nervous system, symbolism attempts to represent and simulate the mind with symbolic systems. All roads lead to the same purpose and jointly study and simulate biological intelligence.

In 2005, in On Intelligence, Hawkins proposed a "memory -prediction model" as the mechanism of cognitive and intellectual activities in the human cerebral cortex. It is considered that the cerebral cortex contains at least 6 neuron-layers with different structures and functions. Where does creativity come from? "The memory-prediction framework of intelligence can provide some answers and useful insights. [28]"

Nevertheless, Hawkins also asserts that existing AI, including ANN, cannot produce true intelligence because they focus only on behavior. He wrote [28]: "We have to extract intelligence from within the brain. No other road will get us there.""We can now see where Alan Turing went wrong. Prediction, not behavior, is the proof of intelligence." Only by studying the operation of the cerebral cortex can we learn "real intelligence". This reminds us of the idea of "peeling onion" as a way of negating previous AI's thinking. In fact, in AI discipline, it is absurd to deny the value of behavior simulation. In fact, Hawkins's "memory prediction model" is also a behavioral simulation, that is, the simulation of cortical behavior.

If memory and prediction is the main indicator of intelligence, the current AI model is well deserved. For example, a trained ANN remembers the training data model and can predict new events; computer chess, obviously can not do without memory and prediction; The key to the AI formulation system introduced in [23] is memory and knowledge discovery, and the ability to predict the performance of products using new materials.

In recent years, deep learning has achieved remarkable results. Its initiator Hinton, LeCun and Bengio have an open mind, advocate the combination of multiple learning methods and algorithms [29], and promote cross-cutting research in the fields of AI, Internet and brain science. This is very beneficial to the development of AI discipline.

Reasoning 13. There is no insurmountable gap between human intelligence and AI.

A popular way to deny the feasibility of AI is to point out directly that some human intelligent behavior $b(i, \mathrm{x})$ is impossible for a machine to simulate. That is to say, $b(i, \mathrm{x})$ is an insurmountable gap between human intelligence and AI. Historically, this gap has been: writing poetry, drawing, playing chess, translation, learning, knowledge discovery and so on.

According to the "simulation feasibility hypothesis", $b(i, \mathrm{x})$ should be unknown, but the "hypothesis of the existence of unknown factors" points out that it is not an eternal mystery, it can be gradually transformed into known. The purpose of science is to achieve this transformation and make it possible to simulate it. The "simulation feasibility hypothesis" points out that simulation is feasible as long as the "gap" is described concretely and accurately, and the "gap" is crossed. Therefore, a fixed "insurmountable gap" does not exist.

In the course of AI development, there have been one after another "gap" has been straddled. For example, in 1960s, AI skeptics said, computers can not play chess at all. After E. Shannon's success in computer checkers (1956), the "gap" in the 1980s was changed to chess, the "most brilliant manifestation of human intelligence". After losing the 1997 world chess championship, the "gap" was redefined as the "mysterious" Chinese Go. In 2006, they were still laughing at the fact that "computer Go is so clumsy". In 2018, AlphaGo shook the world by an absolute margin and gracefully crossed the last gap in chess.

As the "onion skin" metaphor vividly reveals, unwilling doubters continue to reset the gap and find new mysteries such as comprehension $[18,23]$, intentionality [31], consciousness [25], incalculability and quantum uncertainty [25]. However, scientists are getting more and more clear knowledge about these subjects, the simulation of them is becoming more and more possible, and the gaps have been retreating repeatedly, and have become something in the picture again and again.

The idea that there is an insurmountable gap between human intelligence and AI is usually related to the mysticism and agnosticism of human spirit. Some even believe in the never-known "soul" and use it to explain the spirit, leaving the foothold of scientific research.

Next, the understanding, intention and consciousness of the machine are further analyzed.

\section{Machine Understanding}

In Casti's essay [23], Wittgenstein insists that human 
thought and language cannot be understood by any non-human being, and therefore machines must not be able to think. Searle's famous thought experiment, the Chinese Room, also tries to prove that even if a computer system can talk to people smoothly, it doesn't understand language at all [11]. Similarly, many people believe that, despite the amazing achievements of computer chess, it is in fact a false name. It played chess yet didn't understand chess. It didn't win by being smarter than a human; it won by being millions of times faster than a human $[2,25]$.

As you know that there is no universally accepted definition of understanding in academia. Let's take Go as an example and ask that does AlphaGo understand Go?

A: Of course. I know rules and know strategies. Chess has reached 9 paragraph.

Q: AlphaGo also knows the rules, knows the strategies, and plays chess more than any human player.

A: It depends on fast calculation, not understanding.

$Q$ : In order to illustrate your understanding of Go, you set three criteria: know rules, strategy and chess skills. Why are these criteria not practical for machines, and what else do we need to confirm whether it has "understanding"?

A: I don't know how to answer this question. Perhaps there are deeper spiritual world problems to be explored.

Logical thinking should be: although I don't know exactly what you understand about Go, I admit you have a deeper understanding based on your behavior and the level of 9 paragraph. Similarly, Ke Jie admits that AlphaGo has a unique and superb understanding of Go, and it has found a completely new Go strategy that is different from humans, and no one knows what that is. G. Kasparov, the world chess champion, made a similar statement, saying he had encountered an "alien intelligence" [3]: The computer can foresee the long-term chess sequence in its decision-making; the machine's refusal to take a step has a short-term decisive advantage in chess, showing a very similar human sense of danger.

Some people say that there is a convincing intelligent behavior machine without "real intelligence"; AlphaGo, who conquered all the human masters, had no understanding of Go (or Deep Blue to chess), that is obvious logical confusion, which is very harmful to the development of AI discipline. Their "understanding" and human chess players have a common (convergence), that is, adhere to the rules and pay attention to strategy; there are also significant specificity (alienation), such as, the way to achieve the goal is different.

Some philosophers insist on the mystery of human understanding and hold that "understanding can not be measured by external behavior." But no one pointed out that if it is not based on behavior, it should be based on what measure to understand. If someone says he understands chess very well, he doesn't know how to do it (external behavior). Wouldn't it be absurd?

Understanding usually refers to people's cognition of things In the face of complex things, people can not reach a complete understanding, it is difficult to achieve a consistent understanding of each other, there is only a "certain degree" or "in a sense" of understanding. Psychologist S. Blackmore emphasized: "No one of us can expect to 'fully understand consciousness,' and I'm not sure what it might be. [32]" Using the terms of Bayesian confirmation theory, it can continuously be improved the Bayesian reliability or reliability of one's own "understanding" and the probability of it never equals 1 . Therefore, no one can say that he fully understands "human thought and language". At present, computer systems can communicate with people smoothly, and machine translation has reached a fairly high level. It must be admitted that it has a "certain sense" or "a certain degree" of understanding of the language.

\section{Intentionality and Consciousness of Machines}

In recent years, the idea that "intentionality" can not be simulated is very popular in philosophy. Philosopher Searle said that the mind is marked by intentional state, which is inherent in the brain's endogenous biochemical role, and therefore can not be simulated by a computer [19]. This argument implies that all brain functions and intelligent behavior cannot be simulated, because they can be called "intrinsic features of brain biochemistry". This argument obviously fails to grasp the essence of "simulation".

Simply put, intentionality mainly refers to people's conscious desire, intend to, wish and with the external world things related (pointing) to the psychological content and ability. For example, you see something, believe in something, expect something, fear something, want something, etc. This is distinguished from unintentional states without any reference, such as nervous, tired, happy, depressed, etc. [33]. Intentionality is an ancient and modern research field. In the past 30 years, intentionality has become a hot topic of interdisciplinary research and interaction, such as brain science, computer science, psychology, linguistics, cognitive science, etc.

Further research and simulation of intention, intentionality and consciousness will enhance AI level. According to the definition of simulation and the hypothesis of simulation feasibility, as long as one has some clear knowledge of intentionality, we can put aside its unknown mystery and start simulation of intentionality temporarily. Modern neurophysiology holds that intentionality and consciousness are the behavior of the brain, that is, the emergence of a complex neuronal system cleverly organized. Intentions, which represent human perception, attention (attention, attraction) and reference (marked with symbols), can be simulated. For example, the attractor dynamics of modern complexity theory can be used to simulate "attention" [33].

Modern Robotics has made remarkable progress on the road of simulating human's physical ability and intelligence, shape and mind. For example, Professor Einstein Robot, an American early childhood education machine, is selling for only $\$ 169$ this year, accurate control of 30 artificial facial muscles has been achieved; hundreds of facial expressions, such as joy, sorrow, fear, confusion, etc., can be identified, and 
intentional attention to and identification of the host, inference of age and gender, as well as the ability to interact with and talk with the expression. This involves perception, recognition, emotional expression, intentionality, consciousness, or part of consciousness.

As the development of cognitive science, neuroscience, language, AI, analytical philosophy and other subjects, the research on mind has developed to an unprecedented level. F. Crick (1916-2004), a British biophysicist and one of the discoverers of DNA structure and Nobel Prize winner, wrote: "Just as we now understand the functions of DNA, RNA, and proteins, most of the mystery of embryology disappears, so will some of the mysterious properties of consciousness after understanding the mechanisms by which consciousness is produced." In the future, if a machine "can try to explain why it makes a choice" and "can decide its own behavior like a person, that is, have an image of itself," then the machine will have "free will. [31]"

\section{Conclusion}

The controversy surrounding the relationship between biological intelligence and AI is often related to confusion. First of all, there is a lack of clear understanding of the nature of convergence and alienation of simulation and simulation intelligence (AI), and they do not agree with the objective fact that the commonalities and differences coexist (Both/And). Some people describe the relationship between biometric intelligence and AI with "exactly the same" and "duplication". Others, on the contrary, call life phenomena "impossible to simulate", deny commonness by difference, reject simulated intelligence by "true intelligence", or oppose "strong AI" to "weak AI", adopt "either true or false"; and simplify Either/Or thinking formula of "either identical or totally nothing".

Secondly, some people seem to acknowledge the achievements of AI on the one hand, and on the other hand strive to establish an "insurmountable gap" between AI and BI, or fall into the "peeling onion skin" logic trap, repeatedly declaring that AI results are simple, mechanical calculations or operations, the "skin" away from the mysterious of life. They went back to the idea that intelligence, understanding, intentionality, consciousness, or mind could not be simulated. Obviously, this attitude violates the essence of science and tends to mysticism. Scientific truth is relative and goes higher in exploration. In addition to correcting some mistakes, new science never abandons what has already been achieved. Modern physics, for example, never claims that relatively "simple" Newtonian physics is not "real" physics, nor that it is the "skin" that should be discarded.

As Turing said: "We can only see a short distance ahead, but we can see plenty there that needs to be done. [1]" This article expects to form a new consensus on the basis of eliminating some wrong concepts and logical confusion, so as to better explore the mysteries of intelligence, consciousness and life, and develop a better AI.

\section{References}

[1] Turing A M. Computing machinery and intelligence [M]. Oxford University Press on behalf of MIND, 1950, 59(236): 433-460.

[2] Boden M A. The Philosophy of Artificial Intelligence [M]. Oxford: Oxford University Press, 1990.

[3] Russell S. Norvig P. Artificial Intelligence: A Modern Approach [M]. Person Education, Inc., publishing as Prentice-Hall, 1995.

[4] Feng T J. Intelligent Machine and Human Being. Science Press (in Chinese) (1983).

[5] Tian-Jin Feng, Ning Yang and Lin-Tao Ma. The Simulation Theory of Intelligence. 2012 Third International Conference on Theoretical and Mathematical Foundations of Computer Science (ICTMF2012), Lecture Notes in Information Technology, Vol. 38: 188-194

[6] Gerrans P. The theory of mind module in evolutionary psychology [J]. Biology and Philosophy, 2002, 17: 305-321.

[7] Bennett M, Dennett D, Hacker P, Searle J. Neuroscience and Philosophy: Brain, Mind, and Language [M]. Columbia University Press, New York, 2007.

[8] Wiener N. Cybernetics: or Control and Communication in the Animal and the Machine [M]. Cambridge, MP: MIT Press, 1948.

[9] Pierre Baldi, Soren Brunak. Bio-informatics-The Machine Learning Approach [M]. Arts \& Licensing International. Inc. USA. 2003.

[10] Von Neumann J. The computer and the brain [M]. New Haven CT, Yale University Press, 1958.

[11] Newell A, Simon H A. Computer Simulation of Human Thinking [J]. Science, 1961, 134(3495): 2011-2017.

[12] Ziman J. edited. Technological Innovation as an Evolutionary Process. [M]. Cambridge University Press, Cambridge, 2000.

[13] Wittgenstein L. Philosophical Investigations. [M]. Oxford: Basil Blackwell, Publisher, 1953.

[14] Hebb D O. The organization of behavior; a neuropsychological theory [M]. Wiley-Interscience, New York, 1949.

[15] Rosenblatt F.: The perceptron: a probabilistic model for information storage and organization in the brain $[\mathrm{J}]$. Psychological Review, 1958, 65: 386-408.

[16] Feng T J. How The Neural Networks Estimate Parameters from Input Values? [J]. In: IEEE International Joint Conference on Neural Networks, I, 1992: 41-46.

[17] Ziman J. Real Science - What It Is, and What It Means [M]. Cambridge University Press, Cambridge, 2000.

[18] Searle J. Minds, brains, and programs [J]. Brain and Behavioral Sciences, 1980, 3: 417-457.

[19] Searle J. The Mystery of Consciousness [M]. A New York Review Book, 1997.

[20] Feng T J, Ma L T, Ding X Q, Yang N, Xiao X Z. Intelligent techniques for cigarette formula design [J]. Mpthmptics and Computers in Simulation, 2008, 77(5-6): 476-486. 
[21] David Gameza, Progress in machine consciousness [J]. Consciousness and Cognition, 2008, 17: 887-910.

[22] Zelazo P D, et al. eds. The Cambridge Handbook of Consciousness [M]. Cambridge University Press, 2007.

[23] Casti J. L. The Cambridge Quintet: A Work of Scientific Speculation [M]. Sphere Books Ltd, 1998.

[24] Kasparov G., The Chess Master and the Computer [J]. The New York Review of Books, 2010, 57(2): 11.

[25] Penrose R. The Emperor's New Mind - Concerning Computers, Minds, and The Laws of Physics [M]. Oxford University Press, 1989.

[26] Chalmers D. Explaining Consciousness: The Hard Problem MP [M]. MIT Press, 1997.

[27] Chalmers D. Philosophy of Mind: Classical and Contemporary Readings [M]. Oxford University Press, 2002.
[28] Hawkins J, Blakeslee S, On Intelligence [M]. Times Books, Henry Holt and Co 2005. www. OnIntelligence.org

[29] LeCun Y, Bengio Y, Hinton G. Deep learning [J]. Nature, Vol 521, Macmillan Publishers Limited, 28 May 2015.

[30] Dreyfus H L. What Computers Still Can't Do: A Critique of Artificial Reason [M]. Cambridge, MIT press 1992.

[31] Crick F. The Astonishing Hypothesis - The Scientific Search for the Soul [M]. Charles Scribner's Sons, 1994.

[32] S. Blackmore, Consciousness: An Introduction [M]. Oxford University Press, 2003, 2010.

[33] Mainzer K. Thinking in Complexity - The Complex Dynamics of Matter, Mind and Mankind [M]. Springer - Verlag Berlin Heidelberg, 2011. 